Revista peruana de biología 26(3): 369 - 378 (2019) doi: http://dx.doi.org/10.15381/rpb.v26i3.XXXX ISSN-L 1561-0837; elSSN: 1727-9933

Universidad Nacional Mayor de San Marcos

\section{Ácidos grasos en cepas de microalgas del Banco de Germoplasma de Organismos Acuáticos del Instituto del Mar del Perú (IMARPE)}

\author{
Fatty acids in microalgae strains from Banco de Germoplasma \\ de Organismos Acuáticos of IMARPE, Peru
}

*Autor para correspondencia

HHA: hhernandez@imarpe.gob.pe

LFR: Iflores@imarpe.gob.pe

ARS: aruiz@imarpe.gob.pe

\author{
Hanna Hernández Acevedo ${ }^{1 *}$, Leenin Flores Ramos ${ }^{2}$, Anthony \\ Ruiz Soto ${ }^{2}$
}

Instituto del Mar del Perú, Área Funcional de Investigaciones en Acuicultura. Perú.
Otros datos de los autores / biografía:

1 HHA: Banco de Germoplasma de Organismos Acuáticos

2 LFR: Laboratorio de Análisis Instrumental

2 ARS: Laboratorio de Análisis Instrumental

ORCID HHA: 0000-0002-2706-0834

ORCID LFR: 0000-0002-6099-4321

ORCID ARS: 0000-0003-3865-3632

Citación:

Hernández Acevedo H., L. Flores Ramos, A. Ruiz Soto. 2019. Ácidos grasos en cepas de microalgas del Banco de Germoplasma de Organismos Acuáticos del Instituto del Mar del Perú (IMARPE). Revista peruana de biología 26(3): 369 - 378 (Septiembre 2019). doi: http://dx.doi.org/10.15381/ rpb.v26i3.XXXX

Palabras clave: diatomeas; clorófitas; dinoflagelados; ácido eicosapentaenoico (EPA); ácido docosahexaenoico (DHA); acuicultura; ácidos grasos; Bioprospección de microalgas.

Keywords: diatoms; chlorophytes; dinoflagellates; eicosapentaenoic acid (EPA); docosahexae-noic acid (DHA); aquaculture; Fatty acid; Microalgae bioprospecting.

\section{Resumen}

Se determinó la composición de ácidos grasos de 54 cepas microalgales colectadas del Perú y mantenidas en el Banco de Germoplasma de Organismos Acuáticos (IMARPE) con la finalidad de determinar su uso nutricional en la acuicultura. Para ello se realizaron cultivos en un volumen de $50 \mathrm{~mL}$ y se determinaron los porcentajes relativos de ácidos grasos mediante transesterificación directa y cromatografía de gases. En el grupo Chlorophyta las microalgas que presentaron los mayores valores de porcentaje relativo de ácidos grasos fueron Chlamydomonas reinhardtii (16:0; $41.2 \%)$, Scenedesmus obtusus (18:1n-7; 33.6\%), Acutodesmus dimorphus (18:1n-9; $37.1 \%)$, Desmodesmus armatus (18:3n-3; 32.2\%) y Tetraselmis contracta (16:4n-3; $16.5 \%)$. En cambio en el grupo Bacillariophyta, los ácidos grasos más abundantes fueron 16:1n-7 en Chaetoceros didymus (20.2\%), 18:4n-3 en Navicula sp. (28.3\%) y EPA en Asterionellopsis sp. (31.5\%). Por otro lado, en el grupo Dinophyta, todas las cepas superaron el $20 \%$ de DHA, en particular, la cepa de Akashiwo sanguinea presentó el mayor porcentaje relativo de este ácido graso $(29.9 \%)$ y de los ácidos grasos 16:0 (24.8\%) y EPA (16\%). Se discute el uso de estas cepas según su contenido de ácidos grasos.

\section{Abstract}

In This work, we determinate the fatty acids composition and their nutritional value in 54 microalgal strains, collected from Peru and stored in Banco de Germoplasma de Organismos Acuáticos (IMARPE). The cultures were grown to $50 \mathrm{~mL}$ and analyzed by direct transesterification and gas chromatography. In the Chlorophyta group, the microalgaes that present the highest relative percentage of fatty acids were Chlamydomonas reinhardtii (16:0; 41.2\%), Scenedesmus obtusus (18:1n-7; 33.6\%), Acutodesmus dimorphus (18:1n-9; 37.1\%), Desmodesmus armatus (18:3n-3; 32.2\%) and Tetraselmis contracta $(16: 4 n-3 ; 16.5 \%)$. Moreover in the Bacillariophyta group, the most abundant fatty acids were 16:1n-7 in Chaetoceros didymus (20.2\%), 18:4n-3 in Navicula sp. (28.3\%) and EPA in Asterionellopsis sp. (31.5\%). By the other hand, in the Dinophyta group, all strains exceed the $20 \%$ of DHA, in particular Akashiwo sanguinea, it was strain to have the highest percentage of this fatty acid $(29.9 \%)$ in addition to $16: 0(24.8 \%)$ and EPA (16\%). We discussed uses of these strains is according to their fatty acids content. 


\section{Introducción}

Las microalgas son organismos fotosintéticos clasificados en una gran variedad de grupos taxonómicos por sus características morfológicas, fisiológicas y la formación de biocompuestos como proteínas, pigmentos, carbohidratos, vitaminas y ácidos grasos (Patil et al. 2005).

Los estudios en la acuicultura están orientados a la supervivencia larvaria y a la búsqueda y desarrollo de nuevas especies tanto como para cultivo como fuente alimenticia sin dejar de ser una actividad sostenible en el tiempo (Bengtson 2003). Durante años, se han propuesto diferentes especies de microalgas con potencial valor nutritivo para acuicultura, sin embargo esta relación varía dependiendo del origen de la cepa y sobre todo las condiciones de cultivo (Enright et al. 1986). Los géneros de microalgas más utilizados en acuicultura son: Chaetoceros, Thalassiosira, Tetraselmis, Isochrysis, Nannochloropsis, Pavlova y Skeletonema siendo utilizadas en la alimentación de larvas de peneidos, crustáceos filtradores y peces (Thompson et al. 1993). El valor nutricional de una dieta en base de microalgas se encuentra relacionado con la habilidad de satisfacer los nutrientes esenciales, el tamaño apropiado para la ingesta y su digestibilidad (Brown et al. 2002). Uno de los requerimientos es la calidad de los lípidos suministrados como alimento, especialmente los ácidos grasos esenciales poliinsaturados de cadena larga (Pillay 1997), por ejemplo la familia de los omega 3 (20:5n-3, ácido eicosapentaenoico (EPA) y 22:6n-3n, ácido docosahexaenoico (DHA)). Estos son considerados esenciales para el desarrollo adecuado de los organismos cultivables (Patil et al. 2005), dado que intervienen en la formación larvaria y aumenta la capacidad de supervivencia de estas (Whyte 1987).

Muchos organismos del zooplancton e invertebrados marinos dependen de la ingesta de microalgas como único alimento durante todo el su ciclo de vida (Muller-Feuga 2000). A su vez, el zooplancton es utilizado como vector para la alimentación de larvas de peces por su tamaño y movimiento, lo que incentiva la búsqueda y caza en estadios tempranos (Mihalitsis \& Bellwood 2017). En el caso del cultivo de crustáceos pequeños como la Artemia, el suministro de DHA es esencial durante la metamorfosis de los nauplios y poseen la capacidad de reconversión de DHA en EPA (Han et al. 2000). Por otro lado, el cladócero Daphnia pulex, comúnmente llamada "pulga de agua", posee la capacidad de producir ácidos grasos de cadena larga (EPA y 20:4n-6, ácido araquidónico (AA)) a partir de la ingesta de ácidos grasos de cadena corta (18:3n-3 y 18:2n-6) en condiciones de bajas temperaturas para mantener la fluidez y función de sus membranas celulares ante el estrés físico (Schlechtriem et al. 2006). En invertebrados marinos, existen estudios relacionados a la nutrición mediante la ingesta de diatomeas debido a que son fuente principal de EPA (Dunstan et al. 1994), principalmente en cultivos de ostras donde la ingesta neta de estas microalgas aumenta la supervivencia larvaria y la fijación de estas a un sustrato (Coutteau \& Sorgeloos 1992, Su et al. 1996).

Los peces marinos requieren en su dieta una proporción definida de PUFAs (ácidos grasos poliinsaturados), haciendo énfasis en DHA, EPA y AA. Mientras que los peces de agua dulce son capaces de producir EPA y DHA sintetizado de 18:3n-3; ALA (Sargent et al. 1995). Estos ácidos grasos se les consideran esenciales e importantes para diversas funciones fisiológicas de los organismos como el mantenimiento integral de las membranas celulares y precursores de hormonas eicosanoides (Sargent et al. 1999). En particular una alta proporción de DHA es determinante para la buena formación de órganos visuales en larvas de peces (Navarro et al. 1995). Sin embargo, una dieta excesiva en omega 3 reduce la capacidad reproductiva de los peces afectando el sistema endocrino (Izquierdo et al. 2001) y causa deformidades óseas (Cahu et al. 2003).

Existen biotecnologías para producir EPA y DHA de de microalgas generadoras de PUFAs (Khozin-Goldberg et al. 2011). Los ácidos grasos esenciales no sólo son parte importante en la nutrición de organismos cultivables, sino que ácidos grasos de cadena larga, como el EPA, también interfieren con el crecimiento de ciertas bacterias patógenas como las del género Vibrio, perjudiciales en la formación y desarrollo de larvas y generadoras de enfermedades (Benkendorff et al. 2005, Smith 2010).

En el presente trabajo se informa de la composición de ácidos grasos de 54 cepas microalgales colectadas del Perú, cultivadas en el Banco de Germoplasma de Organismos Acuáticos (Instituto del Mar del Perú) y se discute el uso potencial de estos, como alimento nutritivo para primeros estadios de vida de organismos cultivables.

\section{Materiales y métodos}

Cultivo de microalgas.- Se seleccionaron 54 cepas de microalgas marinas y dulceacuícolas de la colección del Banco de Germoplasma de Organismos Acuáticos, Instituto del Mar del Perú, para realizar cultivos de 50 $\mathrm{mL}$ a partir de un inóculo de $10 \mathrm{~mL}$ con una densidad celular aproximada de 1000 células/ mL. Los cultivos fueron mantenidos en cámaras climáticas bajo las siguientes condiciones: las cepas marinas utilizaron el medio F/2 Guillard con silicatos y las microalgas dulceacuícolas usaron el medio CHU modificado, temperatura de $17 \pm 1$ ${ }^{\circ} \mathrm{C}$, pH $7 \pm 1$, intensidad lumínica de $35 \pm 5 \mu \mathrm{mol} . \mathrm{s}^{-1} \cdot \mathrm{m}^{-2} \mathrm{y}$ fotoperiodo 12:12 (horas luz: oscuridad) por un periodo de 10 días. El último día se realizó el conteo de la densidad celular de los cultivos con una cámara Neubauer y un microscopio óptico. La Tabla 1 muestra la lista de cepas con sus respectivas especies y densidades celulares.

Extracción y Análisis de ácidos grasos.- Los cultivos fueron centrifugados a $4500 \mathrm{rpm}$ por 15 minutos a $10{ }^{\circ} \mathrm{C}$ para obtener el pellet y se determinó el contenido de los ácidos grasos con el método de transesterificación directa (Griffiths et al. 2010), el método consiste en la saponificación de los lípidos con metóxido de sodio, seguido de la metilación de sus respectivos ácidos grasos con una solución de trifloruro de boro en metanol para producir los metilésteres y ser analizados por cromatografía gaseosa en el equipo Varian CP-3800, usando una columna Restek, Stabilwax® WCOT fused silica $30 \mathrm{mx} 0.25 \mathrm{mmx} 0.25 \mu \mathrm{m}$, método de inyección splitlles ( 0.50 minuto), volumen de inyección de $1 \mu \mathrm{L}$, temperatu- 
Tabla 1. Cepas de microalgas del Banco de Germoplasma de Organismos Acuáticos del IMARPE y densidades celulares analizadas.

\begin{tabular}{|c|c|c|c|}
\hline Phylum & Código de cepa & Especie & Densidad celular ( $\times 10^{5}$ células $/ \mathrm{mL}$ ) \\
\hline \multirow{32}{*}{ Chlorophyta } & IMP- BG- 003 & Tetraselmis contracta & 6.58 \\
\hline & IMP- BG- 018 & Tetraselmis suecica & 4.48 \\
\hline & IMP- BG- 055 & Tetraselmis suecica & 11.01 \\
\hline & IMP- BG- 277 & Chlamydomonas reinhardtii & 2.94 \\
\hline & IMP- BG- 219 & Pediastrum boryanum & 8.50 \\
\hline & IMP- BG- 255 & Pediastrum boryanum & 10.31 \\
\hline & IMP- BG- 288 & Pediastrum cf. oientale & 5.40 \\
\hline & IMP- BG- 171 & Comasiella arcuata & 19.75 \\
\hline & IMP- BG- 176 & Desmodesmus sp. & 11.69 \\
\hline & IMP- BG- 194 & Desmodesmus communis & 14.53 \\
\hline & IMP- BG- 195 & Acutodesmus dimorphus & 2.46 \\
\hline & IMP- BG- 205 & Acutodesmus dimorphus & 52.25 \\
\hline & IMP- BG- 215 & Scenedesmus obtusus & 17.00 \\
\hline & IMP- BG- 249 & Scenedesmus breviaculeatus & 6.54 \\
\hline & IMP- BG- 253 & Desmodesmus sp. & 15.88 \\
\hline & IMP- BG- 254 & Desmodesmus subspicatus & 8.45 \\
\hline & IMP- BG- 259 & Desmodesmus armatus & 4.46 \\
\hline & IMP- BG- 262 & Desmodesmus armatus & 13.99 \\
\hline & IMP- BG- 265 & Desmodesmus armatus & 15.03 \\
\hline & IMP- BG- 275 & Desmodesmus armatus & 17.20 \\
\hline & IMP- BG- 285 & Desmodesmus armatus & 12.95 \\
\hline & IMP- BG- 287 & Desmodesmus armatus & 12.49 \\
\hline & IMP- BG- 306 & Desmodesmus brasiliensis & 11.68 \\
\hline & IMP- BG- 377 & Desmodesmus dispar & 13.20 \\
\hline & IMP- BG- 382 & Desmodesmus communis & 17.45 \\
\hline & IMP- BG- 389 & Desmodesmus armatus & 16.09 \\
\hline & IMP- BG- 393 & Desmodesmus armatus & 9.98 \\
\hline & IMP- BG- 394 & Desmodesmus armatus & 13.43 \\
\hline & IMP- BG- 402 & Desmodesmus brasiliensis & 15.58 \\
\hline & IMP- BG- 406 & Desmodesmus armatus & 6.79 \\
\hline & IMP- BG- 408 & Desmodesmus armatus & 52.25 \\
\hline & IMP- BG- 423 & Desmodesmus subspicatus & 87.50 \\
\hline \multirow{16}{*}{ Bacillariophyta } & IMP- BG- 057 & Chaetoceros socialis & 0.68 \\
\hline & IMP- BG- 056 & Chaetoceros debilis & 5.60 \\
\hline & IMP- BG- 058 & Chaetoceros compressus & 4.15 \\
\hline & IMP- BG- 059 & Chaetoceros lorenzianus & 1.04 \\
\hline & IMP- BG- 369 & Chaetoceros constrictus & 0.44 \\
\hline & IMP- BG- 435 & Chaetoceros didymus & 1.48 \\
\hline & IMP- BG- 433 & Chaetoceros sp. & 1.30 \\
\hline & IMP- BG- 097 & Asterionellopsis sp. & 2.04 \\
\hline & IMP- BG- 364 & Skeletonema var. maiori & 2.04 \\
\hline & IMP- BG- 434 & Skeletonema costatum & 9.69 \\
\hline & IMP- BG- 365 & Navicula sp. & 0.54 \\
\hline & IMP- BG- 432 & Gyrosigma sp. & 0.15 \\
\hline & IMP- BG- 428 & Thalassiosira sp. & 0.21 \\
\hline & IMP- BG- 436 & Thalassiosira sp. & 0.14 \\
\hline & IMP- BG- 431 & Entomoneis alata & 0.28 \\
\hline & IMP- BG- 054 & Stephanopyxis turris & 0.59 \\
\hline \multirow{6}{*}{ Dinophyta (Miozoa) } & IMP- BG- 036 & Prorocentrum micans & 0.12 \\
\hline & IMP-BG- 062 & Prorocentrum micans & 0.002 \\
\hline & IMP- BG- 116 & Prorocentrum micans & 0.009 \\
\hline & IMP- BG- 115 & Prorocentrum gracile & 0.14 \\
\hline & IMP- BG- 112 & Scippsiella trochoidea & 0.17 \\
\hline & IMP- BG- 127 & Akashiwo sanguinea & 0.001 \\
\hline
\end{tabular}

ra del inyector fue de $250^{\circ} \mathrm{C}$, el gas de arrastre fue helio a $1 \mathrm{~mL} / \mathrm{min}$, la rampa de temperatura fue de $120^{\circ} \mathrm{C}$ por un minuto, $30{ }^{\circ} \mathrm{C} / \mathrm{min}$ hasta $160{ }^{\circ} \mathrm{C}, 160^{\circ} \mathrm{C}$ por un minuto, $4^{\circ} \mathrm{C} / \mathrm{min}$ hasta $240^{\circ} \mathrm{C}, 240{ }^{\circ} \mathrm{C}$ por 7 minutos. El tiempo total del programa de temperatura fue de 30.33 min y la temperatura programada del detector de $260{ }^{\circ} \mathrm{C}$.

Análisis Estadístico.- Los datos fueron analizados en el programa estadístico $\mathrm{R}$ y ordenados mediante funciones de clusters (PCA y Heat Map). Los ácidos grasos fueron agrupados en ácidos grasos saturados (SAFAs), ácidos grasos monoinsaturados (MUFAs) y ácidos grasos poliinsaturados (PUFAs).

\section{Resultados}

Composición de ácidos grasos de 54 cepas de microalgas.- La Tabla 2 representa la composición de ácidos grasos identificados del primer grupo de 16 microalgas Chlorophyta. Los ácidos grasos que presentaron mayor valor de porcentaje relativo en este grupo fueron $16: 0$ (41.2\%), 18:1n-7 (33.6\%), 18:1n-9 (37.1\%), 18:3n$3(32.2 \%)$ y $16: 4 n-3(16.5 \%)$ en Chlamydomonas rein- 
hardtii, Scenedesmus obtusus, Acutodesmus dimorphus, Desmodesmus armatus y Tetraselmis contracta respectivamente. En este grupo la cepa $C$. reinhardtii se diferencia del resto porque no se detectó el ácido graso 18:4n-3 y registró un 15\% más del ácido graso 16:0. Las cepas del género Tetraselmis (clorófitas marinas) presentaron mayores valores de EPA dentro del grupo. Los SAFAs obtuvieron valores entre 10 y $50 \%$ y el mayor aporte fue del ácido graso 16:0. Los dos valores más altos de $₫ 3$ los presentaron T. suecica (50.5\%) y D. armatus (48.7\%) y el valor más bajo lo presentó $C$. reinhardtii $(7.8 \%)$, el mayor aporte fue del ácido graso 18:3n-3. El valor más alto de $₫ 6$ lo presentó la cepa IMP-BG-219, $P$. boryanum (10.2\%), y el mayor aporte fue del ácido graso 18:2n-6.

En la Tabla 3 se agruparon las cepas del género Desmodesmus del grupo Chlorophyta. Los ácidos grasos de las cepas de $D$. armatus presentaron diferentes valores a pesar que son de la misma especie. Los ácidos grasos que presentaron mayor porcentaje fueron 16:0 (26.1\%), 18:1n-7 (32.4\%) y 18:3n-3 (26.8\%) en la cepas IMP-BG-262, IMPBG-265 e IMP-BG-285 respectivamente. Este género presentó un porcentaje de EPA que no sobrepasó el $0.6 \%$ y no se detectó presencia de DHA. Los SAFAs obtuvieron valores entre 16 y $26 \%$ y el mayor aporte fue del ácido graso 16:0 al igual que el grupo anterior. Los MUFAs obtuvieron valores entre 20 y $40 \%$ y el mayor aporte fue del ácido graso $18: 1 n-7$ y $18: 1 n-9$. El valor más alto de $₫ 3$ lo presentó la cepa IMP-BG-275 de la especie D. armatus (47.1\%) y el mayor aporte fue del ácido graso 18:3n-3 mientras que en $₫ 6$ fue del ácido graso 18:2n-6.
La Tabla 4 muestra los ácidos grasos de las especies del grupo Bacillariophyta, comúnmente denominadas diatomeas. Los ácidos grasos más abundantes fueron el 14:0, 16:1n-7, 18:1n-7 y EPA. En este grupo las cepas del género Chaetoceros mostraron un alto valor de 14:0 y 18:1n-7 en comparación a otros géneros, siendo $C$. didymus la especie con mayor porcentaje (31.5\%) de 14:0 y C. compressus en 18:1n-7 (24.8\%). Por otro lado, la cepa de Skeletonema var. mariori presentó el mayor porcentaje en 16:1n-7 (20.2\%), mientras que la cepa de Navicula sp. mostró el mayor porcentaje de 18:4n-3 (28.3\%). Este último ácido graso se registró en poca cantidad y muchas veces no se detectó en el grupo del género de Chaetoceros. Todas las cepas del grupo Bacillariophyta registraron altos valores de EPA, siendo Asterionellopsis sp. la especie con mayor porcentaje de este ácido graso (31.5\%) seguida de Chaetoceros debilis (31.3\%). La cepa que obtuvo el valor más alto de PUFAs (45.5\%) y de $\odot 3$ (44.5\%) fue Navicula sp. siendo el EPA el ácido graso de mayor aporte.

En la Tabla 5 se agruparon las cepas del grupo Miozoa o Dinophyta las cuales presentaron altos valores de DHA superando el $20 \%$ en todas las cepas analizadas. Akashiwo sanguinea fue la especie que presentó mayor porcentaje de este ácido graso (29.9\%) además de 16:0 (24.8\%) y 20:5n-3 (EPA) (16\%). Sin embargo en comparación con las otras especies de este grupo, A. sanguinea presentó un porcentaje menor a $1 \%$ del ácido graso $\mathrm{C} 18: 5 \mathrm{n}-3$, siendo la cepa IMP-BG-062 de la especie P. micans la que obtuvo mayor porcentaje en este ácido graso (26.9\%). Los SAFAs presentaron valores entre el 20 y $30 \%$ y el mayor aporte

Tabla 2. Composición de ácidos grasos del grupo Chlorophyta (grupo $N^{\circ} 1$ ). (a) T. contracta, (b) T. suecica, (c) T. suecica, (d) C. reinhardtii, (e) P. boryanum, (f) P. boryanum, (g) P. cf. orientale, (h) C. arcuata, (i) D. armatus, (j) D. communis, (k) A. dimorphus, (I) A. dimorphus, (m) S. obtusus, (n) S. breviaculeatus, (o) D. armatus, (p) D. subspicatus. Resultados expresados en porcentaje relativo.

\begin{tabular}{|c|c|c|c|c|c|c|c|c|c|c|c|c|c|c|c|c|}
\hline FA & (a) & (b) & (c) & (d) & (e) & (f) & (g) & (h) & (i) & (j) & (k) & (I) & (m) & (n) & (o) & (p) \\
\hline $14: 0$ & 0.4 & - & 0.6 & 4.6 & 0.3 & 0.2 & 0.2 & 1.2 & 0.4 & 0.7 & - & 0.3 & 0.4 & - & 0.6 & 1.3 \\
\hline $16: 0$ & 23.5 & 22.1 & 25.8 & 41.2 & 16.6 & 15.0 & 14.7 & 25.9 & 24.2 & 20.6 & 21.7 & 21.1 & 28.9 & 26.1 & 11.3 & 19.3 \\
\hline 18:0 & 0.4 & - & 0.5 & 1.0 & 0.3 & 0.2 & 0.4 & 2.1 & 2.4 & 3.2 & 3.0 & 1.5 & 2.4 & 1.7 & 1.3 & 1.9 \\
\hline ¿SAFAs & 24.3 & 22.1 & 26.9 & 46.8 & 17.2 & 15.4 & 15.3 & 29.2 & 27.0 & 24.5 & 24.7 & 22.9 & 31.7 & 27.8 & 13.2 & 22.5 \\
\hline $16: 1 n-7$ & 0.4 & 0.6 & 1.4 & 3.9 & 2.1 & 0.4 & 2.4 & 1.3 & 0.8 & 1.7 & 0.6 & 0.3 & 0.5 & 2.2 & 0.8 & 3.3 \\
\hline $16: 1 n-9$ & 5.5 & 7.9 & 3.3 & 9.2 & 4.4 & 9.2 & 6.5 & 7.0 & 6.1 & 3.6 & 0.4 & 5.7 & 3.9 & 1.5 & 6.5 & 3.0 \\
\hline $18: 1 n-7$ & 3.3 & 4.3 & 22.6 & 18.3 & 32.7 & 2.0 & 2.8 & 3.3 & 20.4 & 1.5 & 1.2 & 30.7 & 33.6 & 5.9 & 1.7 & 30.6 \\
\hline $18: 1 n-9$ & 7.1 & 6.7 & - & 3.5 & - & 20.3 & 22.5 & 11.0 & - & 7.3 & 37.1 & - & - & 15.1 & 15.7 & - \\
\hline ¿MUFAs & 16.3 & 19.5 & 27.3 & 34.9 & 39.2 & 31.9 & 34.2 & 22.6 & 27.3 & 14.1 & 39.3 & 36.7 & 38.0 & 24.7 & 24.7 & 36.9 \\
\hline $16: 2 n-4$ & 0.3 & 0.9 & 0.1 & - & 4.0 & - & 3.3 & - & - & - & - & - & - & - & - & - \\
\hline $16: 3 n-4$ & - & - & - & - & - & - & - & - & - & - & - & - & - & - & - & - \\
\hline $16: 4 n-3$ & 16.5 & 14.9 & 10.7 & 2.0 & 3.8 & 5.0 & 7.3 & 5.9 & 6.1 & 11.9 & 3.1 & 6.7 & 3.4 & 5.3 & 10.9 & 4.0 \\
\hline $18: 2 n-6$ & 3.3 & 3.8 & 5.4 & 3.5 & 5.1 & 8.7 & 5.6 & 5.6 & 6.6 & 4.9 & 8.4 & 6.5 & 4.6 & 4.5 & 5.7 & 9.2 \\
\hline $18: 3 n-3$ & 16.3 & 14.2 & 13.9 & 5.8 & 17.7 & 26.4 & 22.3 & 22.2 & 21.3 & 21.3 & 1.0 & 15.7 & 12.3 & 23.2 & 32.2 & 16.9 \\
\hline $18: 3 n-6$ & 0.6 & 1.2 & 0.3 & 2.6 & 0.4 & 0.3 & 0.3 & - & 1.1 & 1.2 & - & 0.5 & - & 0.4 & 0.7 & 0.4 \\
\hline $18: 4 n-3$ & 10.4 & 12.7 & 4.9 & - & 2.6 & 1.8 & 3.4 & 9.1 & 5.0 & 5.2 & 2.6 & 3.8 & 5.3 & 4.2 & 4.9 & 3.0 \\
\hline $18: 5 n-3$ & 0.9 & 1.8 & 1.6 & - & 2.6 & 0.9 & 0.8 & - & 0.7 & 0.2 & 0.5 & 0.7 & 0.2 & 0.9 & 0.3 & - \\
\hline $20: 3 n-3$ & - & - & - & - & - & - & - & - & 0.3 & 1.4 & 3.6 & - & - & 1.0 & 0.4 & - \\
\hline $20: 4 n-3$ & 0.9 & - & 0.5 & - & - & 0.3 & - & - & - & - & 1.0 & 0.7 & - & - & - & - \\
\hline $20: 4 n-6$ & 0.7 & 0.4 & 0.4 & - & 0.3 & 1.2 & 0.6 & - & 0.1 & 0.5 & 1.3 & 0.8 & - & - & - & - \\
\hline $20: 5 n-3$ & 5.5 & 2.4 & 4.9 & - & 0.2 & 0.2 & - & - & - & 1.6 & 0.5 & - & 0.4 & - & - & - \\
\hline $22: 5 n-3$ & - & - & - & - & - & - & - & - & - & - & - & - & - & - & - & - \\
\hline $22: 6 n-3$ & - & - & - & - & 0.9 & 1.1 & 0.6 & 1.3 & 0.1 & - & - & - & 1.1 & - & - & - \\
\hline EPUFAs & 55.4 & 52.3 & 42.7 & 13.9 & 37.6 & 45.9 & 44.2 & 44.1 & 41.3 & 48.2 & 22.0 & 35.4 & 27.3 & 39.5 & 55.1 & 33.5 \\
\hline DHA/EPA & - & - & - & - & 4.5 & 5.5 & - & - & - & - & - & - & 2.8 & - & - & - \\
\hline$\Sigma D H A+E P A$ & 5.5 & 2.4 & 4.9 & - & 1.1 & 1.3 & 0.6 & 1.3 & 0.1 & 1.6 & 0.5 & - & 1.5 & - & - & - \\
\hline ద3 & 50.5 & 46.0 & 36.5 & 7.8 & 27.8 & 35.7 & 34.4 & 38.5 & 33.5 & 41.6 & 12.3 & 27.6 & 22.7 & 34.6 & 48.7 & 23.9 \\
\hline ద6 & 4.6 & 5.4 & 6.1 & 6.1 & 5.8 & 10.2 & 6.5 & 5.6 & 7.8 & 6.6 & 9.7 & 7.8 & 4.6 & 4.9 & 6.4 & 9.6 \\
\hline ค3/ద6 & 11.0 & 8.5 & 6.0 & 1.3 & 4.8 & 3.5 & 5.3 & 6.9 & 4.3 & 6.3 & 1.3 & 3.5 & 4.9 & 7.1 & 7.6 & 2.5 \\
\hline
\end{tabular}


Tabla 3. Composición de ácidos grasos del grupo Chlorophyta (grupo $N^{\circ} 2$ ). (a) D. armatus, (b) D. armatus, (c) D. armatus, (d) D. armatus, (e) D. armatus, (f) D. armatus, (g) D. brasiliensis, (h) D. dispar, (i) D. communis, (j) D. armatus, (k) D. armatus, (I) D. armatus, (m) D. brasiliensis, (n) D. armatus, (o) D. armatus, (p) D. subspicatus. Resultados expresados en porcentaje relativo.

\begin{tabular}{|c|c|c|c|c|c|c|c|c|c|c|c|c|c|c|c|c|}
\hline FA & (a) & (b) & (c) & (d) & (e) & (f) & (g) & (h) & (i) & (j) & (k) & (I) & $(m)$ & (n) & (o) & (p) \\
\hline $14: 0$ & 1.7 & 1.0 & 1.1 & - & - & - & 0.1 & 0.4 & 1.0 & 2.6 & - & - & 0.2 & - & 0.1 & 0.1 \\
\hline $16: 0$ & 19.3 & 21.0 & 26.1 & 18.0 & 24.3 & 25.2 & 24.5 & 20.4 & 16.9 & 20.2 & 22.2 & 20.6 & 16.5 & 21.6 & 15.9 & 15.4 \\
\hline 18:0 & 1.5 & 0.8 & 0.8 & 0.7 & 1.4 & 0.7 & 1.6 & 3.4 & 0.7 & - & 0.5 & 0.4 & 0.8 & 0.6 & 1.2 & 1.1 \\
\hline ¿SAFAs & 22.5 & 22.8 & 28.0 & 18.7 & 25.7 & 25.9 & 26.2 & 24.2 & 18.6 & 22.8 & 22.7 & 21.0 & 17.5 & 22.2 & 17.2 & 16.6 \\
\hline $16: 1 n-7$ & 3.8 & 0.7 & 4.3 & 1.1 & 4.9 & 0.8 & 0.3 & 1.1 & 0.9 & 1.5 & 1.1 & 0.9 & 4.5 & 2.0 & 0.4 & 0.3 \\
\hline $16: 1 n-9$ & 3.4 & 6.1 & 5.2 & 4.1 & 1.5 & 10.7 & 6.9 & 9.9 & 4.3 & 4.9 & 5.8 & 7.1 & 6.2 & 6.1 & 2.4 & 4.0 \\
\hline $18: 1 n-7$ & 22.7 & 1.5 & 3.7 & 4.3 & 32.4 & 1.5 & 26.2 & 2.1 & 23.4 & 4.1 & 2.5 & 1.7 & 2.4 & 2.2 & 21.9 & 22.2 \\
\hline $18: 1 n-9$ & - & 16.0 & 12.5 & 9.3 & - & 15.4 & - & 13.0 & - & 9.9 & 15.7 & 10.9 & 22.0 & 15.3 & - & - \\
\hline EMUFAs & 29.9 & 24.3 & 25.7 & 18.8 & 38.8 & 28.4 & 33.4 & 26.1 & 28.6 & 20.4 & 25.1 & 20.6 & 35.1 & 25.6 & 24.7 & 26.5 \\
\hline $16: 2 n-4$ & - & - & - & - & - & - & - & 0.2 & - & - & - & - & - & - & - & - \\
\hline $16: 3 n-4$ & - & - & - & - & - & - & - & 0.2 & - & - & - & - & - & - & - & - \\
\hline $16: 4 n-3$ & 8.9 & 8.8 & 8.6 & 19.2 & 3.3 & 5.6 & 6.6 & 10.8 & 11.1 & 9.2 & 5.7 & 8.3 & 10.7 & 6.8 & 8.9 & 9.4 \\
\hline $18: 2 n-6$ & 9.4 & 5.8 & 3.5 & 3.8 & 8.4 & 8.1 & 3.7 & 3.9 & 10.7 & 7.9 & 10.1 & 9.7 & 6.5 & 8.9 & 8.6 & 7.7 \\
\hline $18: 3 n-3$ & 18.8 & 26.8 & 12.9 & 16.2 & 11.3 & 14.8 & 16.7 & 13.5 & 16.7 & 23.8 & 16.1 & 22.6 & 16.8 & 19.9 & 25.0 & 24.6 \\
\hline $18: 3 n-6$ & 0.8 & 0.5 & 0.8 & 0.8 & 1.1 & 2.0 & 0.8 & 2.5 & 1.7 & 0.8 & 2.0 & 1.5 & 1.3 & 1.0 & 1.2 & 1.0 \\
\hline $18: 4 n-3$ & 3.4 & 3.6 & 4.4 & 7.2 & 2.8 & 4.7 & 4.5 & 5.3 & 7.4 & 5.8 & 4.7 & 6.1 & 3.7 & 5.2 & 9.0 & 8.6 \\
\hline $18: 5 n-3$ & - & 0.4 & - & - & 0.6 & 0.3 & 1.1 & 0.8 & - & - & 0.3 & - & 0.2 & 0.3 & - & - \\
\hline $20: 3 n-3$ & - & 1.2 & - & 3.2 & - & - & 0.6 & 1.4 & - & - & - & - & - & - & - & - \\
\hline $20: 4 n-3$ & - & - & - & 1.3 & - & - & 0.7 & 0.4 & - & - & 0.7 & - & 0.5 & - & 0.2 & - \\
\hline $20: 4 n-6$ & - & 0.5 & - & - & - & - & - & 0.3 & - & - & - & - & 0.2 & - & 0.2 & 0.1 \\
\hline $20: 5 n-3$ & - & - & - & - & - & 0.5 & - & - & - & - & 0.6 & 0.3 & 0.2 & 0.3 & - & - \\
\hline $22: 5 n-3$ & - & - & - & - & - & - & - & 0.3 & - & - & - & - & - & - & - & - \\
\hline $22: 6 n-3$ & - & - & - & - & - & - & - & - & - & - & - & - & - & - & - & - \\
\hline ¿PUFAs & 41.3 & 47.6 & 30.2 & 51.7 & 27.5 & 36.0 & 34.7 & 39.6 & 47.6 & 47.5 & 40.2 & 48.5 & 40.1 & 42.4 & 53.1 & 51.4 \\
\hline DHA/EPA & - & - & - & - & - & - & - & - & - & - & - & - & - & - & - & - \\
\hline ¿DHA+EPA & 0.0 & 0.0 & 0.0 & 0.0 & 0.0 & 0.5 & 0.0 & 0.0 & 0.0 & 0.0 & 0.6 & 0.3 & 0.2 & 0.3 & 0.0 & 0.0 \\
\hline D3 & 31.1 & 40.8 & 25.9 & 47.1 & 18.0 & 25.9 & 30.2 & 32.5 & 35.2 & 38.8 & 28.1 & 37.3 & 32.1 & 32.5 & 43.1 & 42.6 \\
\hline ద6 & 10.2 & 6.8 & 4.3 & 4.6 & 9.5 & 10.1 & 4.5 & 6.7 & 12.4 & 8.7 & 12.1 & 11.2 & 8.0 & 9.9 & 10.0 & 8.8 \\
\hline ค3/ద6 & 3.0 & 6.0 & 6.0 & 10.2 & 1.9 & 2.6 & 6.7 & 4.9 & 2.8 & 4.5 & 2.3 & 3.3 & 4.0 & 3.3 & 4.3 & 4.8 \\
\hline
\end{tabular}

Tabla 4. Composición de ácidos grasos del grupo Bacillariophyta. (a) C. socialis, (b) C. debilis, (c) C. compressus, (d) C. lorenzianus, (e) C. constrictus, (f) C. didymus, (g) C. sp., (h) A. sp., (i) S. var. maiori, (j) S. costatum, (k) N. sp., (I) G. sp., (m) T. sp., (n) T. sp., (o) E. alata, (p) S. turris. Resultados expresados en porcentaje relativo.

\begin{tabular}{|c|c|c|c|c|c|c|c|c|c|c|c|c|c|c|c|c|}
\hline FA & (a) & (b) & (c) & (d) & (e) & (f) & (g) & (h) & (i) & (j) & (k) & (I) & (m) & (n) & (o) & (p) \\
\hline $14: 0$ & 16.3 & 23.7 & 9.7 & 19.5 & 23.3 & 31.5 & 26.6 & 12.1 & 22.2 & 21.4 & 16.4 & 27.5 & 21.5 & 16.3 & 16.9 & 15.5 \\
\hline $16: 0$ & 11.6 & 6.0 & 15.8 & 8.9 & 1.0 & 19.4 & 13.3 & 6.9 & 5.8 & 8.5 & 19.1 & 12.2 & 6.6 & 22.8 & 14.4 & 24.2 \\
\hline 18:0 & 1.9 & 1.0 & 2.2 & 0.7 & 0.7 & - & 1.4 & 0.6 & 0.6 & - & 0.5 & - & - & - & 0.4 & 1.0 \\
\hline ¿SAFAs & 29.8 & 30.7 & 27.7 & 29.1 & 25.0 & 50.9 & 41.3 & 19.6 & 28.6 & 29.9 & 36.0 & 39.7 & 28.1 & 39.1 & 31.7 & 40.7 \\
\hline $16: 1 n-7$ & 8.5 & 14.0 & 1.0 & 21.0 & 16.6 & 13.1 & 15.1 & 13.3 & 20.2 & 17.0 & 4.3 & 17.1 & 8.5 & 12.6 & 16.8 & 20.4 \\
\hline $16: 1 n-9$ & 1.4 & 0.9 & 6.2 & 3.2 & 6.8 & 4.8 & 7.0 & 3.5 & 1.6 & 4.3 & 3.2 & 2.2 & 3.7 & 4.0 & 3.5 & 3.0 \\
\hline $18: 1 n-7$ & 19.0 & 7.1 & 24.8 & 6.4 & 1.5 & 15.9 & 18.4 & 5.6 & 4.8 & 5.8 & 0.8 & 1.7 & 2.9 & 2.9 & 3.5 & 7.1 \\
\hline $18: 1 n-9$ & 5.0 & 2.6 & 8.9 & 2.3 & 1.4 & 2.1 & 1.2 & 0.7 & 2.6 & 1.5 & 6.2 & 0.6 & 0.8 & 1.1 & 0.6 & 1.1 \\
\hline ¿MUFAs & 33.9 & 24.6 & 40.9 & 32.9 & 26.3 & 35.9 & 41.7 & 23.1 & 29.2 & 28.6 & 14.5 & 21.6 & 15.9 & 20.6 & 24.4 & 31.6 \\
\hline $16: 2 n-4$ & 2.6 & 3.4 & 0.8 & 9.8 & 7.1 & - & 2.7 & 3.1 & 3.7 & 2.5 & 0.3 & 3.4 & 1.5 & 2.7 & 5.2 & 1.5 \\
\hline $16: 3 n-4$ & 1.3 & 1.9 & - & 4.1 & 5.1 & 2.1 & 1.3 & 1.5 & 9.7 & 11.7 & 0.3 & 1.5 & 4.3 & 4.8 & 14.4 & 4.3 \\
\hline $16: 4 n-3$ & 0.7 & 0.2 & - & - & 0.6 & - & - & - & - & 0.4 & 0.4 & 0.3 & - & - & - & - \\
\hline $18: 2 n-6$ & 0.8 & 0.3 & 0.7 & - & 1.9 & - & - & 1.0 & 1.6 & 1.0 & 0.9 & 0.2 & 2.1 & 1.8 & 1.4 & - \\
\hline $18: 3 n-3$ & 1.7 & 1.2 & 4.4 & - & 0.2 & - & - & 1.0 & 0.9 & 0.4 & - & - & - & - & - & - \\
\hline $18: 3 n-6$ & - & 0.2 & - & - & 0.3 & - & - & - & 0.2 & 0.3 & 0.5 & - & - & - & 1.2 & 1.1 \\
\hline $18: 4 n-3$ & - & 0.5 & - & - & 0.7 & - & - & 1.3 & 3.3 & 3.1 & 28.3 & 3.2 & 7.5 & 4.5 & 2.0 & - \\
\hline $18: 5 n-3$ & 0.2 & - & - & - & - & - & - & - & - & - & 0.5 & - & - & - & - & - \\
\hline $20: 3 n-3$ & - & - & - & - & - & - & - & - & - & - & - & - & - & - & - & - \\
\hline $20: 4 n-3$ & - & - & - & - & 0.2 & - & - & 0.5 & - & - & 0.3 & - & - & - & - & - \\
\hline $20: 4 n-6$ & 1.7 & 0.2 & - & 2.1 & 0.4 & - & - & 0.8 & - & - & - & - & - & - & 2.6 & - \\
\hline $20: 5 n-3$ & 6.6 & 31.3 & 4.9 & 14.7 & 11.6 & 6.8 & 6.1 & 31.5 & 14.0 & 12.8 & 14.0 & 14.3 & 23.9 & 14.9 & 5.6 & 12.6 \\
\hline $22: 5 n-3$ & - & - & - & - & - & - & - & - & - & - & 1.0 & - & - & - & - & - \\
\hline $22: 6 n-3$ & - & - & - & 1.8 & 1.2 & - & - & 1.1 & 1.8 & 1.4 & - & 6.6 & 2.1 & 1.6 & 4.3 & 1.1 \\
\hline ¿PUFAs & 15.6 & 39.2 & 10.8 & 32.5 & 29.3 & 8.9 & 10.1 & 41.8 & 35.2 & 33.6 & 46.5 & 29.5 & 41.4 & 30.3 & 36.7 & 20.6 \\
\hline DHA/EPA & - & - & - & 0.1 & 0.1 & - & - & - & 0.1 & 0.1 & - & 0.5 & 0.1 & 0.1 & 0.8 & 0.1 \\
\hline ¿DHA+EPA & 6.6 & 31.3 & 4.9 & 16.5 & 12.8 & 6.8 & 6.1 & 32.6 & 15.8 & 14.2 & 14.0 & 20.9 & 26.0 & 16.5 & 9.9 & 13.7 \\
\hline D3 & 9.2 & 33.2 & 9.3 & 16.5 & 14.5 & 6.8 & 6.1 & 35.4 & 2.0 & 18.1 & 44.5 & 24.4 & 33.5 & 21.0 & 11.9 & 13.7 \\
\hline ద6 & 2.5 & 0.7 & 0.7 & 2.1 & 2.6 & - & - & 1.8 & 1.8 & 1.3 & 1.4 & 0.2 & 2.1 & 1.8 & 5.2 & 1.1 \\
\hline ద3/ద6 & 3.7 & 47.4 & 13.3 & 7.9 & 5.6 & - & - & 19.7 & 11.1 & 13.9 & 31.8 & - & 16.0 & 11.7 & 2.3 & 12.5 \\
\hline
\end{tabular}


Tabla 5. Composición de ácidos grasos de los grupos Miozoa (Dinophyta). (a) P. micans, (b) P. micans, (c) P. micans, (d) P. gracile, (e) $S$. trochoidea, (f) A. sanguinea. Resultados expresados en porcentaje relativo.

\begin{tabular}{|c|c|c|c|c|c|c|}
\hline FA & (a) & (b) & (c) & (d) & (e) & (f) \\
\hline $14: 0$ & 2.4 & 1.9 & 2.1 & 2.4 & 2.0 & 6.7 \\
\hline $16: 0$ & 22.1 & 21.4 & 21.8 & 23.1 & 22.4 & 24.8 \\
\hline $18: 0$ & 1.0 & 1.2 & 1.1 & - & 1.8 & 3.0 \\
\hline ¿SAFAs & 25.5 & 24.5 & 25.0 & 25.5 & 26.2 & 34.5 \\
\hline $16: 1 n-7$ & 1.4 & - & 1.0 & - & 3.3 & 2.6 \\
\hline $16: 1 n-9$ & 1.0 & - & 1.4 & 1.4 & 2.2 & - \\
\hline $18: 1 n-7$ & 3.0 & 1.4 & 1.8 & - & 1.2 & - \\
\hline $18: 1 n-9$ & 1.0 & 1.0 & 1.0 & 1.5 & 2.8 & 4.1 \\
\hline$\Sigma$ MUFAs & 6.4 & 2.4 & 5.2 & 2.9 & 9.5 & 6.7 \\
\hline $16: 2 n-4$ & - & - & - & - & - & - \\
\hline $16: 3 n-4$ & - & - & - & - & - & - \\
\hline $16: 4 n-3$ & - & - & - & 1.1 & - & - \\
\hline $18: 2 n-6$ & 0.8 & - & - & 2.8 & - & - \\
\hline $18: 3 n-3$ & 2.9 & 2.4 & 2.6 & - & 1.3 & - \\
\hline $18: 3 n-6$ & - & - & - & - & - & - \\
\hline $18: 4 n-3$ & 8.0 & 9.6 & 9.4 & 12.9 & 8.6 & 3.0 \\
\hline $18: 5 n-3$ & 19.0 & 26.9 & 23.2 & 14.2 & 15.9 & 1.0 \\
\hline $20: 3 n-3$ & 4.1 & 4.6 & 4.1 & 4.4 & 5.0 & - \\
\hline $20: 4 n-3$ & - & - & - & - & - & - \\
\hline $20: 4 n-6$ & 4.0 & 4.3 & 3.3 & 5.7 & 3.5 & - \\
\hline $20: 5 n-3$ & 1.5 & 1.3 & 1.9 & 1.7 & 5.9 & 16.0 \\
\hline $22: 5 n-3$ & 0.9 & - & - & - & - & - \\
\hline $22: 6 n-3$ & 26.3 & 23.8 & 25.3 & 28.7 & 21.8 & 29.9 \\
\hline ¿PUFAs & 67.5 & 72.9 & 69.8 & 71.5 & 62.0 & 49.9 \\
\hline DHA/EPA & 17.5 & 18.3 & 13.3 & 16.9 & 3.7 & 1.9 \\
\hline$\Sigma \mathrm{DHA}+\mathrm{EPA}$ & 27.8 & 25.1 & 27.2 & 30.4 & 27.7 & 45.9 \\
\hline ద3 & 62.7 & 68.6 & 66.5 & 63.0 & 58.5 & 49.9 \\
\hline ద6 & 4.8 & 4.3 & 3.3 & 8.5 & 3.5 & 0.0 \\
\hline ๑3/ద6 & 13.1 & 16.0 & 20.2 & 7.4 & 16.7 & - \\
\hline
\end{tabular}

fue del ácido graso 16:0 al igual que los grupos anteriores. Los MUFAs presentaron valores bajos en comparación a otros grupos (de 2 a 10\%). Los PUFAs presentaron valores entre los 49 y $72 \%$ siendo el de mayor aporte el ácido graso DHA y 18:5n-3. Los valores de $₫ 3$ estuvieron alrededor de $70 \%$ siendo el DHA el ácido graso de mayor aporte. Los valores de $₫ 6$ estuvieron entre 3 a 9\%, siendo el 20:4n-6 el ácido graso de mayor aporte.

Reconocimiento de grupos taxonómicos.- La Figura 1 agrupa las cepas estudiadas según las características de los ácidos grasos estudiados, también llamados marcadores químico-taxonómicos. Se observa una agrupación según los filos Chlorophyta, Bacillariophyta y Dinophyta. El grupo Chlorophyta presenta 4 ácidos grasos discriminantes (18:3n-3, 16:4n-3, 18:2n-6 y 16:1n-9) al igual que el grupo Bacillariophyta (14:0, EPA, 16:1n-7 y 16:2n-4), mientras que el grupo Dinophyta presenta 3 ácidos grasos discriminantes (DHA, 18:5n-3 y 18:4n-3).

En el Heat Map de la Figura 2 se clasificaron por clusters las cepas según el porcentaje relativo de ácidos grasos. En el dendograma de las cepas se observó dos agrupamientos Dinophyta con Chlorophyta y dejando separado a Bacillariophyta. En el dendograma de los ácidos grasos se observó dos agrupamientos en el cual los ácidos grasos 16:0, 18:3n-3 y 18:1n-9 se encuentran separados en un grupo. Dentro de otro grupo se separan los ácidos grasos EPA, 16:1n-7 y 14:0 del resto. Al relacionar ambos dendrogramas se observó que el grupo Bacilla-

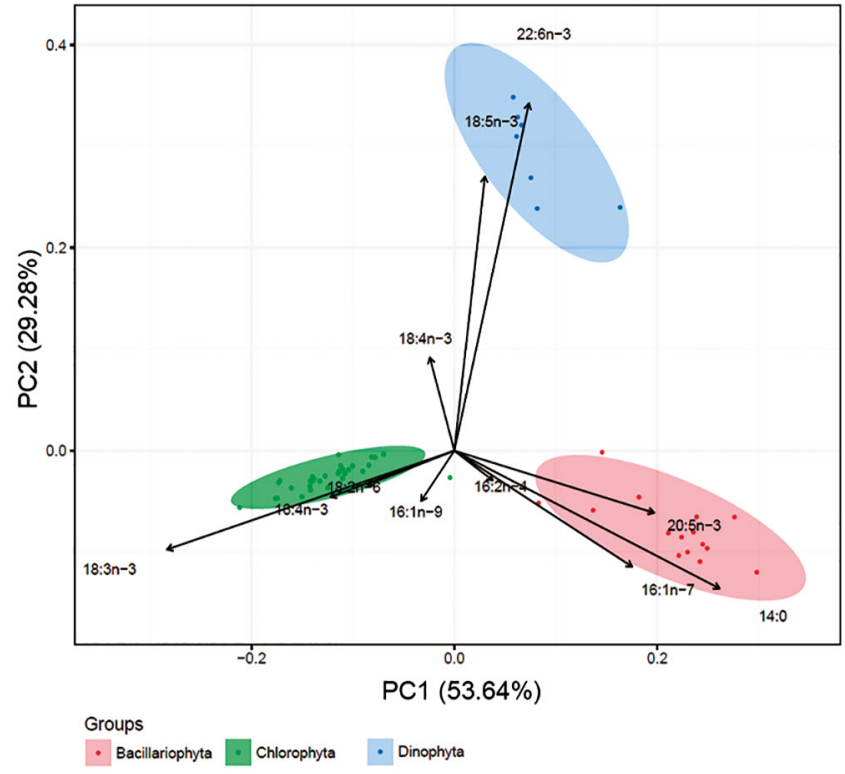

Figura 1. Análisis de Componentes Principales (PCA) agrupando las cepas estudiadas según las características de los ácidos grasos estudiados.

riophyta contiene el clúster con los ácidos grasos EPA, 16:1n-7 y 14:0 más abundantes mientras que el grupo Dinophyta o Miozoa contiene el clúster de los ácidos grasos 18:5n-3 y DHA. El grupo Chlorophyta engloba más variedad de ácidos grasos siendo 16:0, 18:3n-3 y 18:1n-9 los que presentaron mayor proporción en comparación a otras cepas. Ciertos géneros de Chaetoceros del grupo Bacillariophyta se agruparon dentro del clúster del grupo Chlorophyta al presentar mayor proporción del ácido graso 18:1n-7.

\section{Discusión}

Los resultados de los diagramas del PCA y el Heat Map nos muestran ácidos grasos que actúan como discriminantes de los grupos taxonómicos (Phylum), sin embargo dentro de estos grupos existen otras clasificaciones taxonómicas tales como Familia, las cuales pueden variar en la proporción de los ácidos grasos. Dentro del grupo Chlorophyta, el género Tetraselmis, microalga marina perteneciente a la Familia Chlorodendraceae, contiene una baja proporción de ácidos grasos de cadena C20 y C22 (Guedes \& Malcata 2012, Brown et al. 1997). Algunos autores señalan que las microalgas marinas producen mayor cantidad de PUFAs (EPA y DHA) mientras que las microalgas dulceacuícolas producen mayor cantidad de SAFAs y MUFAs (Patil et al. 2007).

Nuestro estudio muestra que los ácidos grasos del grupo Bacillariophyta tienen altos porcentajes de EPA y 16:1n-7, el grupo Dinophyta de 18:4n-3, 18:5n-3 y DHA y son considerados como biomarcadores taxonómicos, resultados semejantes a los encontrados por Kumari et al. (2013). La gran diversidad de los grupos taxonómicos en microalgas se debe al proceso evolutivo y el origen de los plastidios fotosintéticos (Archibald 2005). De acuerdo con la clasificación taxonómica actual, los Bacil- 

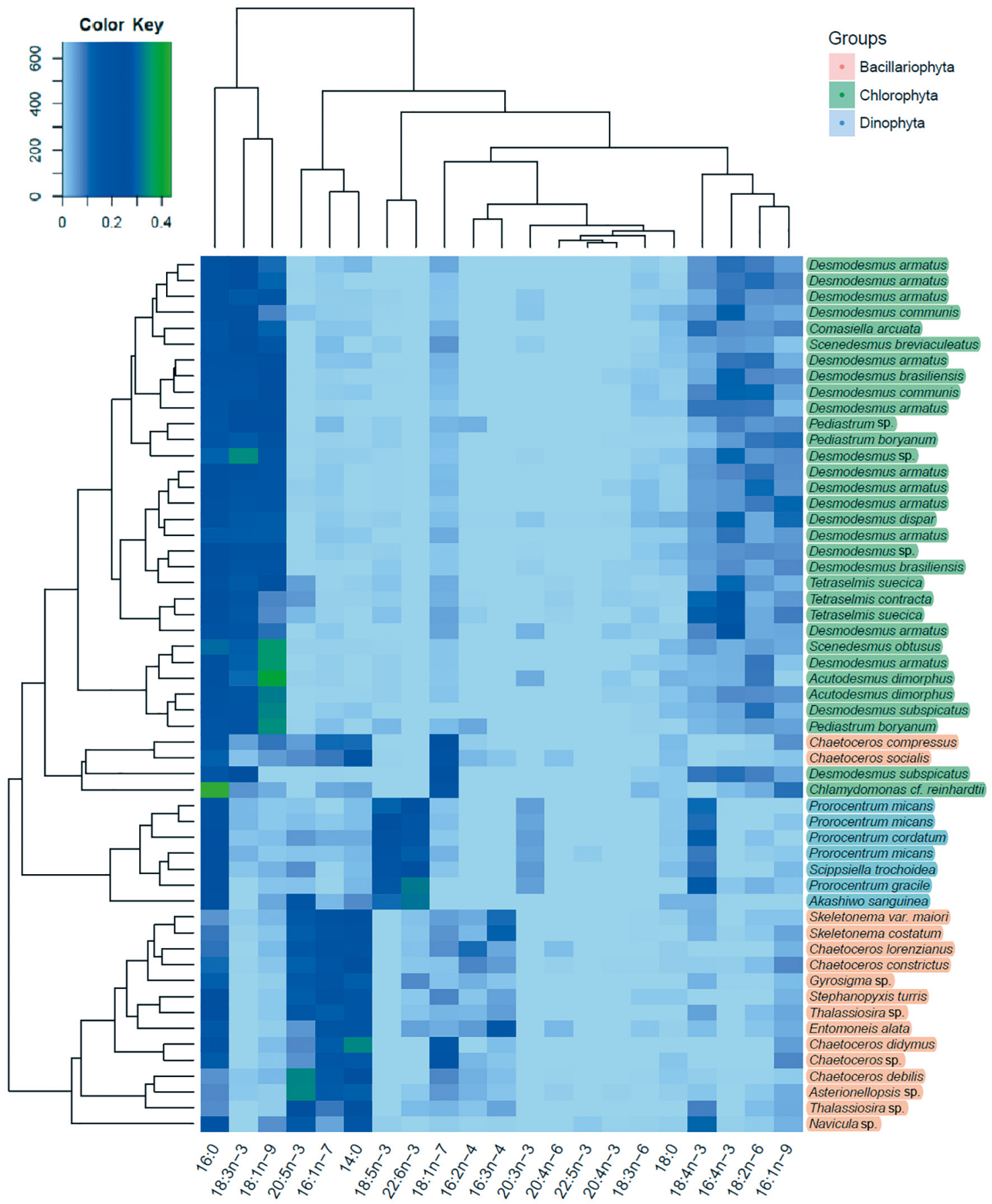

Figura 2. Heat Map de clasificación de cepas caracterizadas por ácidos grasos.

lariophyta y Dinophyta se encuentran emparentados por un ancestro común originario, lo cual explica su capacidad de producir ácidos grasos poliinsaturados de cadena muy larga (Keeling 2010).

También se observaron diferencias en la proporción de ácidos grasos entre cepas de la misma especie. Tal es el caso de las cepas de la especie Desmodesmus armatus, aisladas de diferentes lagunas altoandinas del Perú, que presentaron diferentes proporciones de ácidos grasos aun cuando se cultivaron a las mismas condiciones de laboratorio. Estos resultados concuerdan con lo obtenido por Toledo-Cervantes et al. (2018) en cepas de Scenedesmus obtusiusculus de México, asimismo concu- erdan con los resultados de Selvarajan et al. (2014) en cepas de Chlorella vulgaris de la India. Muchas veces las cepas microalgales de la misma especie pero aisladas de diferentes ambientes exhiben diferentes actividades fisiológicas y producen diferentes biomoléculas (Grama et al. 2014). En estos casos, la diversidad fenotípica (adaptaciones plastidiales de las microalgas) es mayor a la genotípica lo que nos brinda la oportunidad de obtener nuevos metabolitos de diferentes ambientes ecológicos (Thangavel et al. 2018).

De otro lado, Suh et al. (2015) determinaron los perfiles de ácidos grasos de 20 cepas de microalgas marinas de Corea, las cuales 8 especies de microalgas coinciden 
con analizadas en este trabajo (4 de Dinophyta, 3 de Bacillariophyta y 1 de Chlorophyta). En Dinophyta, la cepa de A. akashiwo presentó un mayor porcentaje de DHA superando a la cepa coreana en un $6 \%$, sin embargo presentó un $4.1 \%$ menos de EPA. Por otro lado, las cepas coreanas no identificaron la presencia del ácido graso 18:5n3 (OPA), el cual fue determinado en este análisis con un alto porcentaje en los géneros Prorocentrum y Scrippsiella siendo un ácido graso discriminante para el grupo. También se observó que el porcentaje del ácido graso OPA es 15 veces menor en la cepa $A$. sanguinea en comparación a las demás cepas de este grupo. El ácido graso OPA se encuentra relacionado a los Haptophyta y en muchas microalgas marinas los doble enlaces de este ácido graso están presentes en paredes celulares ubiquitinosas, guardando estrecha relación con la presencia de placas en la pared celular de los géneros Prorocentrum y Scrippsiella. La especie $A$. sanguinea, al ser un dinoflagelado desnudo o con ausencia de tecas, presenta en menor proporción este ácido graso discriminante (Sayanova et al. 2011). En el grupo Bacillariophyta, ambas cepas de la especie $C$. didymus obtuvieron porcentajes parecidos en EPA y 16:0, sin embargo la cepa coreana de $S$. costatum presentó mayor porcentaje en EPA y DHA que la cepa peruana. En el grupo Chlorophyta, la cepa peruana del género Chlamydomonas presentó un mayor porcentaje de 16:0 que la cepa coreana, no obstante, en esta última se determinó la presencia de EPA el cual es discriminante para la clasificación de grupos taxonómicos. Este ácido graso esencial se encuentra muy rara vez en Chlorophyta, siendo algunas especies y cepas que presentan un porcentaje significativo, siendo el caso de $C$. allensworthii (22.3\%) (Lang et al. 2011).

La proporción de ácidos grasos depende del hábito alimenticio de los organismos cultivados. Los peces omnívoros y herbívoros, a lo contrario de los peces carnívoros, poseen la capacidad de sintetizar ácidos grasos de cadena larga (omega 3) mediante la elongación de 18:3n-3 (ALA), por lo tanto requieren una mayor cantidad de este ácido graso y menor cantidad de EPA y DHA en su dieta (Watters et al. 2012). Por lo descrito anteriormente y según los resultados de los análisis de los perfiles de ácidos grasos se sugiere el uso de microalgas clorófitas para la alimentación de peces omnívoros y herbívoros por su alto contenido de ALA y bajo contenido de EPA y DHA. Por otro lado, dentro del grupo Chlorophyta, existen cepas que presentan un alto contenido de $₫ 3$ como Tetraselmis contracta (IMP-BG-003) y Desmodesmus armatus (IMP-BG-259) importantes para la síntesis de ácidos grasos esenciales.

En la acuicultura, los rotíferos se han desarrollado como alimento vivo por la facilidad de su cultivo (Castro et al. 2003), calidad nutritiva (Shepherd \& Bromage 1999) y movimiento adecuado para ser presa fácil (Snell et al. 1987). Sin embargo, el rotífero Brachionus, no presenta la capacidad para producir EPA ni DHA (Whyte \& Nagata 1990). Actualmente los rotíferos son enriquecidos con una dieta basada en microalgas con alto contenido de DHA, como Isochrysis galbana, y así ser utilizados como alimento vivo durante el periodo larval de peces (Watters et al. 2012). También puede enriquecerse a los rotíferos con microencapsulados de aceites y emulsiones, no obstante se recomienda el alimento directo de microalgas por contener vitaminas, proteínas y elementos trazas necesarios para el mantenimiento del organismo (Barclay \& Zeller 1996). Según la relación de ácidos grasos esenciales (DHA/EPA) obtenida en nuestro estudio, las cepas P. micans (IMP-BG-062) y A. sanguinea (IMP-BG-127), del grupo Dinophyta serían una importante fuente de DHA Mientras que las cepas Bacillariophyta E. alata (IMP-BG- 431) y Asterionellopsis sp. (IMP-BG-097), serían importante fuente de EPA. Además, la mayor proporción de $\odot 3 / \infty 6$ (47.4), en la cepa Chaetoceros debilis (IMP-BG- 056) también la indicaría óptima para alimentación de rotíferos. Por otro lado, los cultivos masivos de las especies de Dinophyta estudiadas (P. micans, P. gracile y $S$. trochoidea) presentan un alto contenido de OPA, ácido graso que ha sido reportado como agente hemolítico en el dinoflagelado Karenia mikimotoi durante un episodio de mortandad de krill en las costas de Noruega (Yasumoto et al. 1990).

Durante la etapa larvaria de la concha de abanico $\mathrm{Ar}$ gopecten purpuratus, se les suministra una combinación de diversas microalgas que presentan diferentes proporciones de DHA y EPA. Este último ácido graso es importante en el proceso de la metamorfosis de las larvas a estadios juveniles acelerando el crecimiento del bivalvo (Farías \& Uriarte 2006). En organismos adultos el DHA y el AA son utilizados para la maduración de la gónada y del músculo abductor (Palma-Fleming et al. 2002). Las especies de microalgas más utilizadas para alimentación de estos moluscos son Isochrysis galbana y Chaetoceros calcitrans por la proporción de ácidos grasos (DHA: EPA), mejor digestibilidad y aporte de otros minerales esenciales para la formación y fortalecimiento de valvas (Navarro et al. 2000). Otros autores recomiendan el suministro de microalgas del género Tetraselmis junto con Isochrysis galbana y Chaetoceros calcitrans durante la etapa larval para una mayor supervivencia en el proceso de aparición de mancha ocular entre otros cambios morfológicos (Velasco \& Barros 2008, Uriate et al. 2001, Carreño Hernández et al. 2012). Por lo anteriormente descrito, se recomienda realizar estudios con las cepas Chetoceros debilis (IMP-BG-056) para alimentación de A. purpuratus por su alto contenido de EPA (31.3\%), además de la cepa de P. gracile (IMP-BG-115) por su mayor contenido de AA $(5.7 \%)$ y de DHA $(28.7 \%)$.

\section{Litertaura citada}

Archibald J. 2005. Jumping genes and shrinking genomes-probing the evolution on eukaryotic photosynthesis using genomics. IUBMB Life 57: 539-547. doi: https://doi. org/10.1080/15216540500167732

Barclay W., and S. Zeller. 1996. Nutritional enhancement of n-3 and n- 6 fatty acids in rotifer and Artemia Nauplii by feeding spray- dried Schizochytrium sp. Journal of the World Aquaculture Society 27: 314-322. doi: https:// doi.org/10.1111/j.1749-7345.1996.tb00614.x

Bengtson DA. 2007. Status of Marine Aquaculture in Relation to Live Prey: Past, Present and Future. In: Live Feeds in Marine Aquaculture. John Wiley \& Sons, Ltd. p. 1-16. doi: https://onlinelibrary.wiley.com/doi/ abs/10.1002/9780470995143.ch1. 
Benkendorff K., A.R. Davis, C.N. Rogers, and J.B. Brenner. 2005. Free fatty acids and sterols in the benthic spawn of aquatic molluscs, and their associated antimicrobial properties. Journal of Experimental Marine Biology and Ecology 316: 29-44. doi: https://doi. org/10.1016/j.jembe.2004.10.001

Brown M.R., L.E. Cruz Suárez, D. Ricque Marie, M. Tapia Salazar, M.G. Gaxiola Cortés, N. Simoes. 2002. Nutritional value of microalgae for aquaculture. In Avances en Nutrición Acuícola VI. Memorias del VI Simposium Internacional de Nutrión Acuícola, 60-70. México.

Brown M.R., S.W. Jeffrey, J.K. Volkman, and G.A. Dunstan. 1997. Nutriotional properties of microalgae for mariculture. Aquaculture 151: 315-331. doi: https://doi. org/10.1016/S0044-8486(96)01501-3

Cahu C.H., J.Z. Infante, and T. Takeuchi. 2003. Nutriotional components affecting skeletal development in fish larvae. Aquaculture 227: 254-258. doi: https://doi. org/10.1016/S0044-8486(03)00507-6

Carreño Hernández Y.K., E. Acosta Ortiz, and J. Gómez-León 2012. Crecimiento y supervivencia de larvas de Argopecten nucleus alimentadas con diferentes dietas microalgales. Boletín Investigaciones Marinas y Costeras 41: 103-120. doi: https://doi.org/10.25268/bimc.invemar.2012.41.1.75

Castro T., R. De Lara, G. Castro, J. Castro, and A. Malpica. 2003. Alimento vivo en la acuicultura. ContactoS México 48: $27-33$.

Coutteau P., and P. Sorgeloos. 1992. The use of algal substites and the requirement for live algae in the hatchery and nursery rearing of bivalve molluscs: an international survey. Journal of Shellfish Research 11: 467-476.

Dunstan G.A., J.K. Volkman, S.M. Barrett, J.M. Leroi, and S.W. Jeffrey. 1994. Essential polyunsaturated fatty acids from 14 species of diatom (Bacillariophyceae). Phytochemistry 35: 155-161. doi: https://doi.org/10.1016/ S0031-9422(00)90525-9

Enright C.T., G.F. Newkirk, J.S. Carigie, and J.D. Castell. 1986. Evaluation of phytoplankton as diets for juvenile Ostrea edulis. Journal of Experimental Marine Biology and Ecology 96: 1-13. doi: https://doi.org/10.1016/00220981(86)90009-2

FAO. The State of World Fisheries and Aquaculture 2018. Rome: FAO, 2018.

Farías A., and I. Uriarte. 2006. Nutrition in pectinids. In Scallops: Biology, Ecology and Aquaculture, by S.E. Shumway and G.J. Parsons. 521-542. Elsevier. doi: https://doi. org/10.1016/S0167-9309(06)80036-0

Grama B.S., S. Chader, D. Khelifi, B. Stenuit, C. Jeffryes, and S.N. Agathos. 2014. Characterization of fatty acid and carotenoid production in an Acutodesmus microalga isolated from the Algerian Sahara. Biomass and Bioenergy 69:265-75. doi: https://doi.org/10.1016/j. biombioe.2014.07.023

Griffiths M.J., R.P. Van Hille, and S.T. Harrison. 2010. Selection of direct transesterification as the preferred method for assay of fatty acid content of microalgae. Lipids 45(11): 1053-1060. doi: https://doi.org/10.1007/ s11745-010-3468-2

Guedes A.C., and F. X. Malcata. 2012. Nutriotional value and uses of microalgae in aquaculture. Aquaculture: 5978. doi: https://doi.org/10.5772/30576
Han K., I. Geurden, and P. Sorgeloos. 2000. Comparison of docosahecaenoic acid (22:6n-3) levels in various Artemia strains during enrichment and subsequent starvation. Journal of World Aquaculture Society 31: 469-475. doi: https://doi.org/10.1111/j.1749-7345.2000.tb00897.x

Izquierdo M.S., and H. Fernández Palacios. 2001. Effect of broodstock nutrition on reproductive performance of fish. Aquaculture 197: 25-42. doi: https://doi. org/10.1016/B978-0-444-50913-0.50006-0

Keeling P.J. 2010. The endosymbiotic origin, diversification and fate of plastids. Philosophical Transactions of the Royal Society B 365 (1541): 729-748. doi doi: https:// doi.org/10.1098/rstb.2009.0103

Khozin-Goldberg I., U. Iskandarov and Z. Cohen. 2011. LCPUFA from photosynthetic microalgae: occurrence, biosynthesis, and prospects in biotechnology. Apply Microbiology Biotechnology 91: 905-915. doi: https://doi.org/10.1007/s00253-011-3441-x

Kumari P., M. Kumar, C.R.K. Reddy and B. Jha. 2013. Algal lipids, fatty acids and sterols. In Functional ingredients froam algae for foods and nutraceuticals, by H. Dominquez, 3, 67-76. Woodhead Publishing Ltd: Cambridge. doi: https://doi.org/10.1533/9780857098689.1.87

Lang I., L. Hodac, T. Friedl, and I. Feussner. 2011. Fatty acid profiles and their distribution patterns in microalgae: a comprehensive analysis of more than 2000 strains from the SAG culture collection. BMC Plant Biology 11: 124-140. doi: https://doi.org/10.1186/1471-2229$11-124$

Mihalitsis D.R., and M. Bellwood. 2017. A morphological and functional basis for maximum prey size in piscivorous fishes. Plone One 12 (9): e0184679. doi: https://doi. org/10.1371/journal.pone.0184679.

Muller-Feuga A. 2000. The role of microalgae in aquaculture: situation and trends. Journal of Applied Phycology 12: 527-534. doi: https://doi. org/10.1023/A:1008106304417

Navarro J.C., L.A. McEvoy, M.V. Bell, F. Amat, F. Hontoria and J.R. Sargent. 1995. Effect of dietary lipids on the lipid composition of fish larvae eyes. In Fish and shellfish larviculture symposium. European Aquaculture Society, by P. Lavens, E. Jaspers and E. Roelants, 196-199. Ghent, Belgium: Larvi 95.

Navarro J.M., G.E. Leiva, G. Martinez, and C. Aguilera. 2000. Interactive effects of diet and temperature on the scope for growth of the scallop Argopecten purpuratus during reproductive conditioning. Journal of Experimental Marine Biology and Ecology 247: 67-83. doi: https://doi.org/10.1016/S0022-0981(00)00140-4

Palma-Fleming H., J. M. Navarro, E. Peña, and G. Martínez. 2002. Effect of three conditioning diets on the fatty acid composition of gonads and muscle of Argopcten purpuratus. New Zealand Journal of Marine and Freshwater Research 36(3): 605-620. doi: https://doi.org/10. $1080 / 00288330.2002 .9517116$

Patil V., K.L. Reitan, G. Knutsen, L.M. Mortensen, T. Källqvist, and E. Olsen. 2005. Microalgae as source of polyunsaturated fatty acids for aquaculture. Current Topics in Plant Biology 6: 57-65.

Patil V., T. Kallqvist, E. Olsen, G. Vogt, and H. Gislerod. 2007. Fatty acid composition of 12 microalgae forpossible use in aquaculture feed. Aquaculture International 15: 1-9. doi: https://doi.org/10.1007/s10499-006-9060-3

Pillay T.V.R. 1997. Acuicultura. Principios y prácticas. Ed. Limusa. México: 697. 
Sargent J.R., J.G. Bell, R.J. Henderson, and D.R. Tocher. 1995. Requirement criteria for essential fatty acids. Journal of Applied Ichthyology 11: 183-198. doi: https://doi. org/10.1111/j.1439-0426.1995.tb00018.x

Sargent J., G. Bell, L. McEvoy, D. Tocher, and A. Estevez. 1999. Recent developments in the essential fatty acid nutrition of fish. Aquaculture 177: 191-199. doi: https:// doi.org/10.1016/S0044-8486(99)00083-6

Sayanova O., R.P. Haslam, M.V. Calerón, N.R. López, C. Worthy, and P. Rooks. 2011. Identification and functional characterisation of genes encoding the omega- 3 polyunsaturated fattyh acid biosynthetic pathway from the coccolithophore Emiliania huxleyi. Phytochemistry 72: 594-600. doi: https://doi.org/10.1016/j.phytochem.2011.01.022

Schlechtriem C., M.T. Arts, and I.D. Zellmer. 2006. Effect of temperature on the fatty acid composition and temportal trajetories of fatty acids in fasting Daphnia pulex (Crustacea, Cladocera). Lipids 41: 397-400. doi: https://doi.org/10.1007/s11745-006-5111-9

Selvarajan R., P. Velu, and E. Sanniyasi. 2014. Molecular characterization and fatty acid profiling of indigenous microalgae species with potential for biofuel production in Tamil Nandu India. Internacional Multidisciplinary Research Journal, Golden Reasearch Thoughts 3: 1-9.

Shepherd J., and N. Bromage. 1999. Pisicultura Intensiva. Zaragoza: Acribia S.A.

Smith V.J., A.P. Desbois, and E.A. Dyrynda. 2010. Conventional and unconventional antimicrobials from fish, marine invertebrates and micro-algae. Marine Drugs 8: 12131262. doi: https://doi.org/10.3390/md8041213

Snell T. W., M.J. Childress, and E.M. Boyer. 1987. Assessing the status of rotifer mass culture. Journal World Aquaculture Society 18: 270-277. doi: https://doi. org/10.1111/j.1749-7345.1987.tb01038.x

Su H.M., M.S. Su, and C. Liao. 1996. Preliminary results of providing various combinations of live food to gropuer (Epinephelus coiodes) larvae. In Live Food in Aquaculture: Proceedings of the Live Food and Marine Larviculture Symposium, by A. Hagiwara, T. W. Snell, E. Lubzens and C. S. Tamaru, 301-304. Nagasaki,. doi: https://doi.org/10.1007/978-94-017-2097-7_48

Suh, S., et al. 2015. Fatty acid methyl ester profiles and nutritive values of 20 marine microalgae in Korea. Asian Pacific Journal of Tropical Medicine,: 191-196. doi: https:// doi.org/10.1016/S1995-7645(14)60313-8

Thangavel, Kalaiselvi, et al. 2018. Growth and metabolic characteristics of oleaginous microalgal isolates from Nilgiti biosphere Reserve of India. BMC Microbiology,: 1-17. doi: https://doi.org/10.1186/s12866-017-1144-x

Thompson, P. A, M Guo, and P. J Harrison. 1993. The influence of irradiance on the biochemical composition of three phytoplankton species and their nutritional value for larvae of the Pacific oyster (Crassostrea gigas). Marine Biology 117: 259-268. doi: https://doi.org/10.1007/ BF00345671
Toledo-Cervantes A., G. Garduño Solórzano, J.E. Campos, M. Martínez-García, and M. Morales. 2018. Characterization of Scenedesmus obstusiusculus AT-UAM for highenergy molecules accumulation: deeper insight into biotechnological potential of strains of the same species. Biotechnology Reports 17: 16-23. doi: https:// doi.org/10.1016/j.btre.2017.11.009

Uriate I., G. Rupp, and A. Abarca. 2001. Producción de juveniles de pectínidos iberoamericanos bajo condiciones controladas. In Los moluscos pectínidos de Iberoamérica: ciencia y acuicultura., by A. N. Maeda-Martínez, 501. México: McGraw-Hill Interamericana,.

Velasco L.A., and J. Barros. 2008. Experimental larval culture of the Caribbean scallops Argopecten nucleus and Nodipecten nodosus. Aquaculture 39: 603-618. doi: https://doi.org/10.1111/j.1365-2109.2008.01917.x

Watters C., S. Iwamura, H. Ako, and D-F. Deng. 2012. Nutrition considerations in aquaculture: the importance of omega-3 fatty acids in fishdevelopment and human health. Foods and Nutririon FN-11: 1-7.

Whyte J.N.C. 1987. Biochemical composition and energy content of six species of phytoplankton used in mariculture of bivalves. Aquaculture 119: 231-241. doi: https:// doi.org/10.1016/0044-8486(87)90290-0

Whyte J.N., and W.D. Nagata. 1990. Carbohydrate and fatty acid composition of the rotifer, Brachionusplicatilis, fed monospecific diets of yeast or phytoplankton. Aquaculture 89: 263-272. doi: https://doi. org/10.1016/0044-8486(90)90131-6

Yasumoto T., B. Inderdal, T. Aune, V. Hormazabal, O. M. Skulberg, and Y. Oshima. 1990. Screening for hemolytic and ichthyotoxic components of Chrysochromulina polyepsis and Gyrodinium aureolum from Norwegian coastal waters. In Toxic marine phytoplankton, by E. Graneli, B. Sundstron, L. Edler and D.M. Anderson, 436-440. New York: Elsevier.

\begin{abstract}
Agradecimientos:
Nuestro agradecimiento especial a la Mg. Liz Cecil Tenorio García Blásquez por su colaboración en la entrega de inóculo de cepas del grupo Miozoa (dinoflagelados) y la identificación de estas; al Blgo. Leonardo Mendoza Carbajal por su ayuda en la identificación de especies de las cepas del grupo Chlorophyta.
\end{abstract}

\section{Rol de los autores:}

HHA, LFR, ARS: realizaron el diseño experimental, los experimentos y redactaron el manuscrito; HHA, LFR: realizaron el análisis de datos, revisaron y aprobaron el manuscrito.

Conflicto de intereses:

Los autores no incurren en conflictos de intereses.

Fuentes de financiamiento:

Presupuesto por Resultados: PpR “Ordenamiento y Desarrollo de la Acuicultura" (DGIA- IMARPE).

Aspectos éticos / legales:

Las cepas analizadas son propiedad del Banco de Germoplasma de Organismos Acuáticos del Instituto del Mar del Perú. Permisos de colecta dentro del proyecto: "Búsqueda, caracterización y cultivo de microalgas de zonas altoandinas del Perú potencialmente útiles en la industria cosmética". Convenio de Cofinanciamiento $\mathrm{N}^{\circ}$ : 201-2015-FONDECYT. 